\title{
TUSSOCK BUTTERFLY PROJECT
}

\section{Gavin O'Brien and Hannah Joynt}

\section{INTRODUCTION}

This collaborative design project is the work of two senior lecturers from the Otago Polytechnic College of Art, Design and Architecture, Hannah Joynt and Gavin O'Brien. Together they developed a saleable design product - a reproducible Kiwiana-like' piece of art based on a 1970s New Zealand stamp that features a tussock butterfly. The product not only celebrated the original iconic stamp, but also sought to engage with and contribute to environmental conservation efforts aimed at native New Zealand moths and butterflies. A proportion of the profits from the sale of the product were contributed to conservation project Ahi Pepe I MothNet. This report presents the development and progress of the authors' project.

\section{PROCESS}

Our collaboration began from a casual 'hallway' conversation. We found ourselves discussing a shared interest in a 50-year-old New Zealand postage stamp that we both regard as a somewhat forgotten gem of New Zealand graphic design. After excitedly bouncing

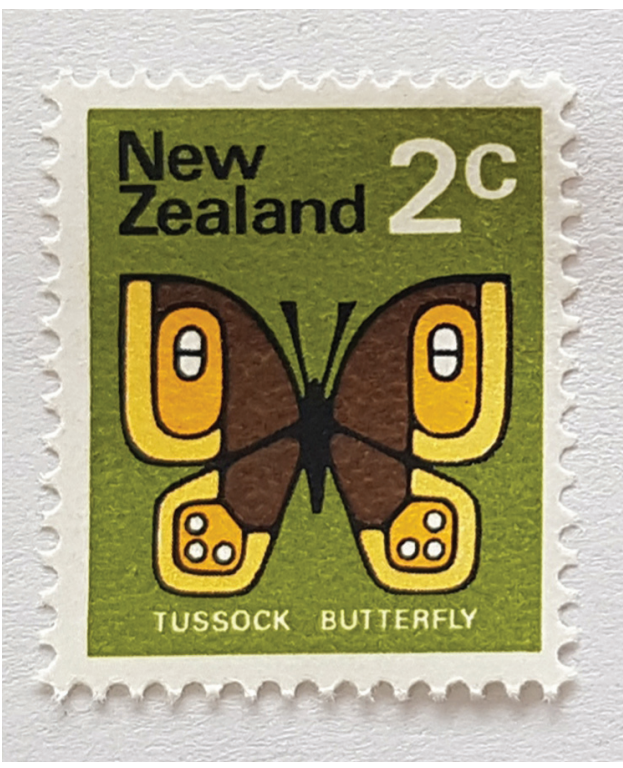

Figure I. Enid Hunter 1970s stamp, designed for New Zealand Post. ideas around on how we might resurrect something of the stamp's former mana, we soon realised that we share similar creative philosophies, interests in environmental conservation and complementary skill sets. We figured that, by working together, we could learn from each other and enjoy continuing discussions that would develop our individual and collaborative creative beliefs and values.

Out of our discussions sprang the question: rather than making art that might 'raise awareness' about an environmental issue, how could we use our art and design practices to contribute more directly to environmental conservation efforts and engage with communities beyond our familiar art and design field? How could our art become a conservation action with a significant ripple effect? From this cocoon of thinking, the Tussock Butterfly project emerged.

The image that sparked it all off was the 1970 New Zealand postage stamp which depicted the tussock butterfly in a stylised modernist manner common to the period. 
The stamp's design was the work of an English immigrant, Enid Hunter, who won the commission as one of a suite of three designs in a 1969 competition run by New Zealand Post. The other two designs in the competition were the one-cent red admiral butterfly stamp and the half-cent stamp depicting the copper glade butterfly.

Our initial intention was simply to translate Hunter's tussock butterfly image into another medium, plywood. We would increase the scale to make it suitable for household display, in a manner that recalled the popular decorative butterfly motif found on the exterior of many New Zealand homes and baches of the 1960s. However, further research on the butterfly's significance - it is endemic to the eastern South Island of New Zealand, with a stronghold in our local coastal Otago area - led us to connect with the conservation project AhiPepe | Mothnet and New Zealand Post. AhiPepe | Mothnet is a citizen science

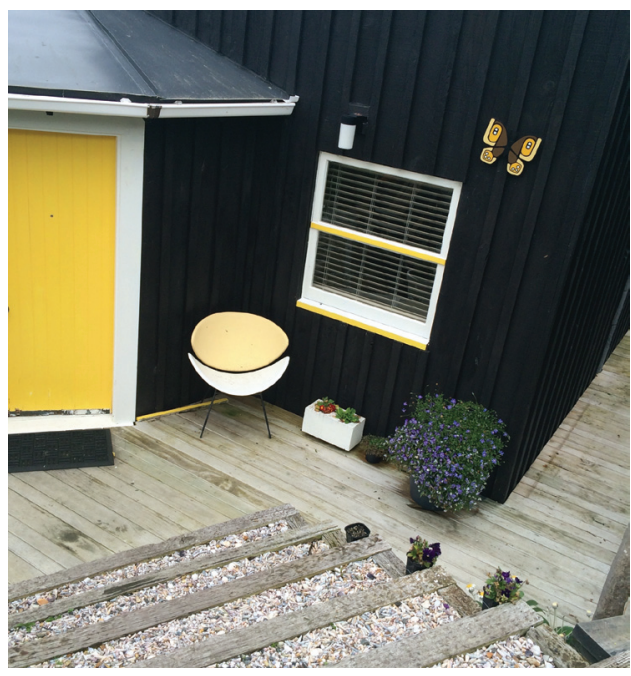

Figure 2. Installed Tussock Butterfly image in context. Image: Gavin O'Brien. project that aims to engage teachers, students and whanau with moths, and through moths with nature and science. ${ }^{2}$ As part of this agenda, the project attempted to raise public appreciation of moths and explore their potential to act as ecological indicators of the health of our natural world. From the outset of this association, we both gained an in-depth understanding of our subject: in particular, we learned that the 'tussock butterfly' is in fact a moth.

With copyright permission from New Zealand Post, we developed a saleable, limited-edition series of 'Tussock Butterflies' based on the stamp design. The product was made with sustainably produced bamboo ply, hand-painted with environmentally friendly paints, and packaged in a custom-made brown cardboard box, together with a brief insert explaining the project. Ten percent of each sale was donated to the AhiPepe | Mothnet project. We have presented the project as a conference paper for the 2019 ITP Whanaungatanga Research Symposium and we are continuing to work with New Zealand Post, with the intention of creating similar designs based on the other two stamps of the series.

We see this design product as an object that braids together many threads: a way for us to use design to contribute and engage with the community and environmental conservation; a celebration of an iconic piece of New Zealand design; a nod to the dwindling hobby of stamp collecting; and a cipher for the changing significance of the postage stamp in the digital age - but also as a powerful way to share the stories signified by our work.

Special thanks are due to Ron Bull, Tumumaki Whakaako, and Barabara Anderson of the AhiPepe Mothnet project for their assistance with this work.

Figure 3. The finished product. Image: Hannah Joynt.

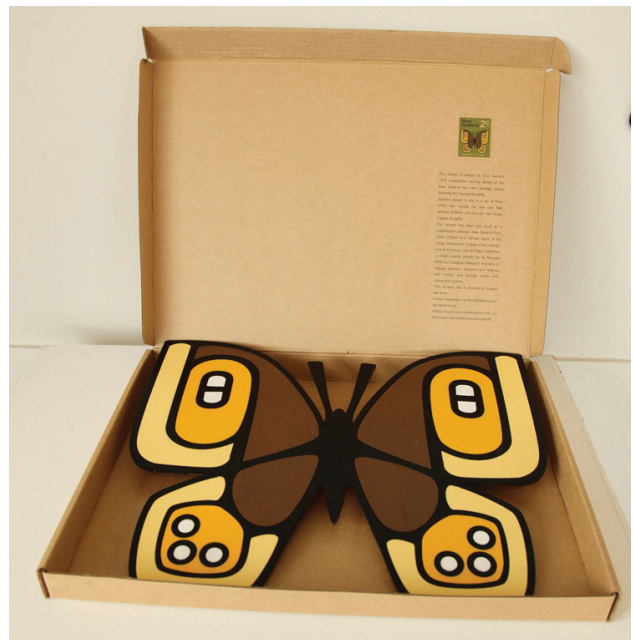




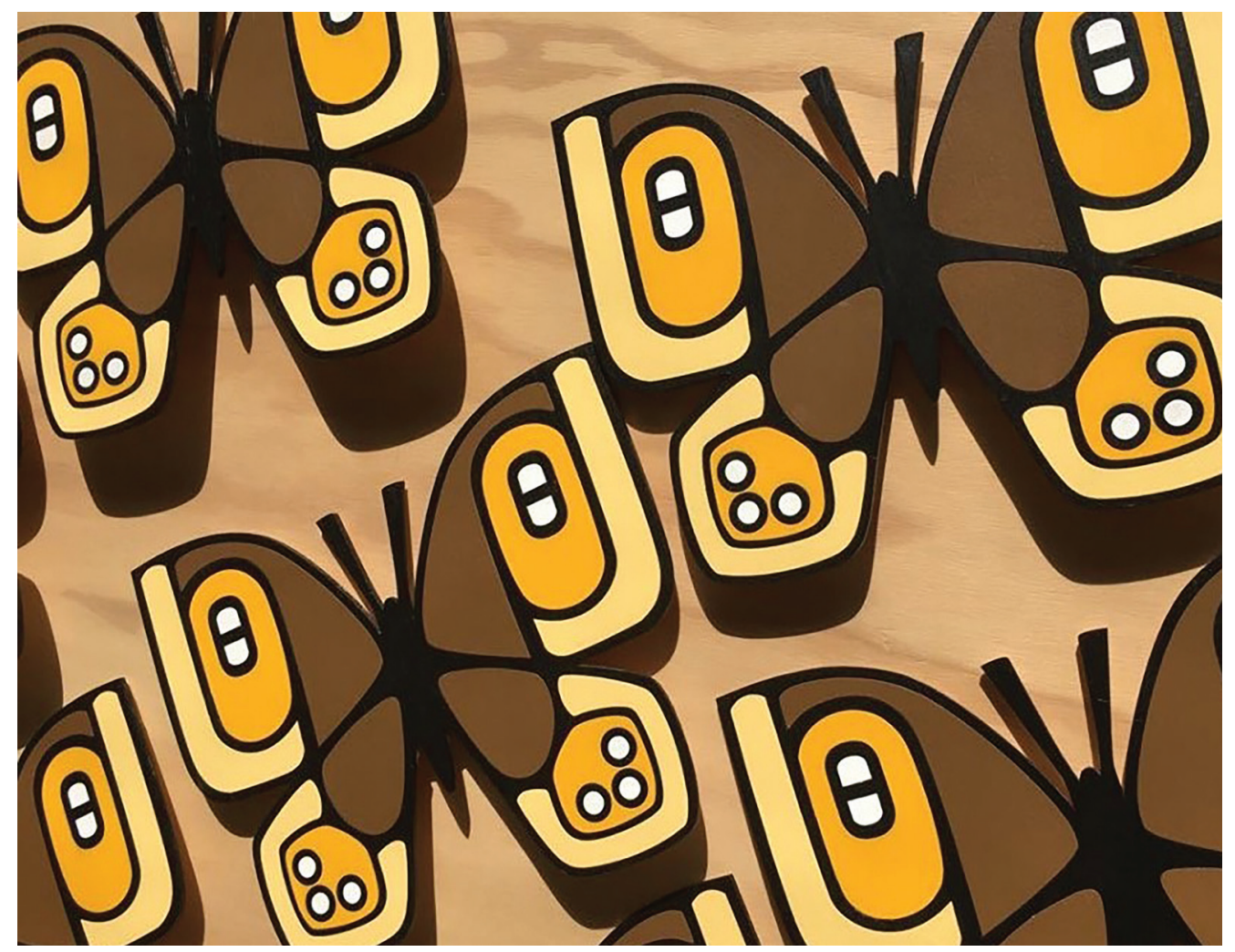

Figure 4. Tussock Butterfly (detail). Image: Hannah Joynt.

Gavin O'Brien is a senior lecturer at Otago Polytechnic College of Art, Design and Architecture. He holds graduate degrees in art, architecture, and applied science and has worked in the fields of architecture and education as well as exhibiting as an artist/designer, where his work explores the common turf between art and design.

Hannah Joynt is a contemporary artist and senior lecturer. She has been teaching at Otago Polytechnic College of Art, Design and Architecture since 2007. Her studio-based art practice is focused on engaging with the landscape and the environment through drawing. She works collaboratively on creative projects that extend her practice into new territories and communities of practice.

I The term Kiwiana refers to certain items relating to New Zealand's cultural heritage, especially from the mid-twentieth century, that are seen as encapsulating iconic New Zealand or Kiwi elements. These "quirky things that contribute to a sense of nationhood" include both genuine cultural icons and kitsch. See https://en.wikipedia.org/wiki/Kiwiana (accessed 20 July 2019).

2 https://mothnet.org/ (accessed 20 July 2019). 